\title{
La composition actuelle de la Société des études robespierristes
}

\section{Michel Biard}

\section{(2) OpenEdition \\ 1 Journals}

Édition électronique

URL : https://journals.openedition.org/ahrf/11133

DOI : 10.4000/ahrf.11133

ISSN : 1952-403X

\section{Éditeur :}

Armand Colin, Société des études robespierristes

\section{Édition imprimée}

Date de publication : 1 septembre 2008

Pagination : 19-27

ISBN : 978-2-200-92515-4

ISSN : 0003-4436

\section{Référence électronique}

Michel Biard, «La composition actuelle de la Société des études robespierristes », Annales historiques de la Révolution française [En ligne], 353 | juillet-septembre 2008, mis en ligne le 01 septembre 2011, consulté le 01 juillet 2021. URL : http://journals.openedition.org/ahrf/11133 ; DOI : https://doi.org/ 10.4000/ahrf.11133

Ce document a été généré automatiquement le 1 juillet 2021.

Tous droits réservés 


\title{
La composition actuelle de la Société des études robespierristes
}

\author{
Michel Biard
}

1 Un siècle après sa naissance, la Société des études robespierristes recense environ 300 abonnés individuels aux AHRF, qui sont en très grande majorité par ailleurs sociétaires 1. En 2005, en prévision de la célébration de ce centenaire, nous avions lancé une enquête auprès des membres de la Société, afin de pouvoir étudier la composition de celle-ci. 133 sociétaires ont alors répondu, ce qui à la fois peut être considéré comme insuffisant, mais permet néanmoins une sorte de « sondage » de bonne qualité sur l'état de la Société. Sur ces 133 sociétaires de 2005, 107 sont des hommes et 26 des femmes, soit environ $80 \%$ d'hommes pour $20 \%$ de femmes. Or, parmi les abonnés à la revue en 2007, 71 sont des femmes, soit une part à peine supérieure (23,5\%), ce qui confirme donc la valeur de notre sondage de 2005. Le caractère minoritaire de cette présence féminine se trouve, en revanche, atténué dans les instances dirigeantes de la Société. Ainsi, au sein du Conseil d'administration actuel, la parité est parfaitement respectée (15 hommes, 15 femmes). Toutefois, il est aisé d'observer, en consultant les noms des présidents successifs de la Société, que jamais une femme n'a accédé à cette responsabilité. Enfin, au sein des actuels comités de rédaction et de lecture des AHRF, il convient également de remarquer un déséquilibre, avec 11 hommes pour 4 femmes, soit un rapport d'environ $73 \%$ pour $27 \%$. Il est bien sûr toujours possible de déplorer ce déséquilibre, $a$ fortiori de chercher à le réduire dans les années à venir, mais force est donc de constater qu'il est le reflet de la composition du groupe des sociétaires et de celui des abonnés aux AHRF.

2 S'agissant de la répartition par âge des sociétaires, nous ne pouvons ici naturellement nous appuyer que sur le sondage de 2005, puisque la Société ne possède pas ce type de renseignement sur chacun de ses membres. Après dépouillement des fiches reçues, 132 des 133 réponses ont livré une date de naissance. Notre benjamin était alors né en 1979, les membres les plus âgés étant nés au début des années 1920, avec deux cas particuliers, ceux de Morris Slavin et de Robert Legrand. Tous deux avaient tenu à répondre à notre enquête et nous ont hélas quitté dans les mois suivants. Robert 
Legrand était né en 1912, Morris Slavin en 1913, c'est-à-dire une décennie avant nos autres sociétaires les plus âgés. La célébration du centenaire de la Société doit aussi être l'occasion de rappeler leur mémoire ${ }^{2}$ et celle de nos autres membres récemment disparus. En 2005, les chiffres obtenus dans cette enquête ne pouvaient que, déjà, nous interpeller sur le vieillissement de la Société (cf. graphique $n^{\circ} 1$ ), et hélas depuis nous avons tous pris deux années de plus et plusieurs de nos membres sont décédés. Environ $24 \%$ de nos membres avaient alors 70 ans et plus et $20 \%$ avaient 60 à 69 ans, soit une part cumulée de $44 \%$. Viennent ensuite deux autres groupes d'âge, $31 \%$ des membres entre 50 et 59 ans, $17 \%$ entre 40 et 49 ans, soit un autre bloc d'importance similaire avec $48 \%$. La suite est évidemment d'une implacable logique : $5 \%$ de membres ont entre 30 et 39 ans, et $3 \%$ moins de 30 ans. Il va de soi que ces chiffres reflètent certaines réalités de la recherche, mais il me semble qu'ils sont aussi le fruit d'un nouvel état du monde depuis les bouleversements de la fin du siècle dernier et les remises en question de la notion même de révolution. En tout état de cause, il s'agit là, d'évidence, d'un constat qui peut paraître inquiétant pour l'avenir même de la Société des études robespierristes. C'est de sa capacité à se renouveler, à s'agréger de nouveaux sociétaires et à susciter des abonnements aux AHRF que la création d'Albert Mathiez pourra continuer à vivre.

3 En bonne logique, les dates d'adhésion à la Société et d'abonnement à la revue épousent en grande partie la structure par âge (cf. graphique $n^{\circ} 2$ ). En 2005, nos deux plus anciens sociétaires et abonnés étaient Robert Legrand (1946) et Morris Slavin (1950), devançant ceux qui les avaient rejoints dans les années 1950 et étaient toujours fidèles à la Société en 2005 : nos deux collègues japonais Michio Shibata (1951) et Haruhiko Hattori (1958), le Norvégien Kâre Tonnesson (1954), l'Américain James Friguglietti (1959), les Français Claude Mazauric (1956), Bernard Plongeron (1956) et Jacques Bourdin (1959) 3 . 18 sociétaires déclaraient avoir rejoint la Société dans les années 1960, 16 autres dans les années 1970, 26 dans les années 1980 avec un impact évident du Bicentenaire (7 pour la seule année 1989) et encore 29 dans les années 1990. Enfin, 27 des sociétaires qui ont répondu à l'enquête de 2005 sont devenus membres de la Société dans la première moitié des années 2000 (2000-2005)4. Même s'il convient naturellement de prendre en compte les disparitions, que ce soit les décès ou le nonrenouvellement d'adhésion, ces chiffres viennent ainsi nuancer le constat précédent et attester que la Société est toujours capable d'attirer à elle de nouveaux membres. Pour s'en convaincre, il suffit à cet égard de consulter les procès-verbaux de ses réunions, régulièrement publiés dans les AHRF.

4 Le profil socio-professionnel de nos sociétaires est, comme chacun pouvait s'y attendre, très nettement marqué par une omniprésence des enseignants (cf. graphique $n^{\circ} 3$ ). Là encore, 132 réponses ont livré la profession du sociétaire, or, si l'on ajoute aux enseignants les chercheurs rattachés au CNRS ou à d'autres organismes, ce groupe représente $77 \%$ des sociétaires. Sans surprise, l'enseignement supérieur est très présent avec 55 membres, mais il convient de se réjouir de la présence parmi nous de 33 enseignants du secondaire et de 2 enseignants du primaire. Les $23 \%$ restants sont si dispersés qu'aucune analyse n'est possible, sauf à livrer un inventaire à la Prévert qui nous donnerait deux officiers, un météorologiste, un biologiste, un cinéaste, un employé de la SNCF, un journaliste, etc. En revanche, les motifs d'adhésion à la Société livrés par ceux qui ont bien voulu répondre à cette question du formulaire d'enquête méritent sans doute qu'on s'y arrête. Comme chacun était libre de donner une ou plusieurs réponses, le corpus s'élève ici à 183 réponses. $59 \%$ d'entre elles soulignent 
une volonté de rejoindre la Société par intérêt pour l'histoire de la Révolution française. Cela paraît bien entendu aller de soi, mais somme toute ce n'est guère qu'une grosse moitié des réponses.

5 Environ $15 \%$ font état de l'influence d'un ou plusieurs membres de la Société, le plus souvent cité étant Albert Soboul, devant Michel Vovelle et divers directeurs de thèse. Force est ici d'espérer que chacun de nos membres qui exerce ce type de responsabilité persiste dans la voie tracée, de manière notamment à ce que des doctorants nous rejoignent, tout particulièrement dans les universités où les recherches sur la période révolutionnaire demeurent importantes. Seules $6 \%$ des réponses évoquent la qualité de la revue publiée par la Société, mais il paraît aller de soi que nombreux sont ceux qui le pensent sans pour autant avoir songé à l'écrire. Enfin, les deux derniers chiffres doivent retenir notre attention : seuls 21 membres $(11,5 \%)$ disent avoir voulu adhérer à une société de pensée et seuls 16 (environ $9 \%$, parfois les mêmes bien sûr) mentionnent la mémoire de Robespierre. Même si un de nos sociétaires se dit « robespierriste depuis l'âge de quinze ans » et qu'un autre évoque une " adhésion militante robespierriste », si quelques-uns évoquent outre la mémoire de l'Incorruptible celle de Saint-Just ou celle de Babeuf, la question posée pour la très grande majorité des sociétaires actuels ne fait néanmoins guère de doute: non pas tant combien de nous se sentent-ils toujours « robespierristes» et avec quels sens donnés à ce mot, mais que signifie le fait d'être membre de cette Société savante un siècle après sa création par Albert Mathiez ? Et, au final, sommes-nous encore réellement une société de pensée? Question pour le moins essentielle quant à l'identité même de la Société des études robespierristes.

6 Le dernier point que je voudrais aborder concerne la répartition géographique de nos membres. Cette fois, je n'ai pas utilisé les chiffres tirés de l'enquête de 2005, mais plus simplement le dernier état de nos abonnés en 2007 (avec donc une légère marge d'erreur puisque, d'une part, quelques abonnés ne sont pas sociétaires, d'autre part, certains ont hélas pris la fâcheuse habitude de régler très tard leurs cotisation et abonnement). Sans guère de surprise, le poids de Paris et de ses banlieues est très important, avec 52 abonnés à Paris et 35 dans les départements environnants, soit quelque $30 \%$ du nombre total. Plusieurs départements et régions sont ensuite des zones de force, le plus souvent en liaison avec des centres universitaires où persiste une tradition souvent ancienne d'études révolutionnaires: 15 abonnés pour les deux départements de Haute-Normandie, dont 12 en Seine-Maritime; 13 dans la région Rhône-Alpes, dont 5 en Isère ; 12 en Bourgogne, dont 8 en Côte-d'Or; 10 dans la région Midi-Pyrénées, dont 8 en Haute-Garonne; 11 dans la région Provence-Alpes-Côte d'Azur, dont 8 dans les Bouches-du-Rhône; 9 également en Auvergne, dont 5 dans le Puy-de-Dôme ; 8 dans le Nord-Pas-de-Calais, avec 4 pour chacun des deux départements de cette région. En revanche, des zones de faiblesse flagrante apparaissent et doivent attirer notre attention dans les années à venir, d'autant qu'il y existe parfois des universités importantes. C'est d'abord le cas du Grand Ouest, de la Bretagne à l'Aquitaine : 6 abonnés seulement dans les quatre départements bretons, 4 pour les cinq départements de la région Pays-de-la-Loire ; 6 pour les quatre départements de PoitouCharentes; 3 seulement pour les cinq départements d'Aquitaine. Sans faire un inventaire exhaustif des régions françaises, c'est également le cas du Nord-Est: 2 abonnés en Alsace ; 3 en Lorraine ; 4 en Franche-Comté. Quant aux abonnés individuels résidant dans des pays étrangers, là encore leur répartition géographique est liée tout à la fois aux héritages des décennies précédentes et aux centres de recherche travaillant sur la période révolutionnaire : 42 en Europe, dont 9 en Italie, 8 en Belgique, 5 au 
Royaume-Uni, 4 en Espagne comme en Allemagne et en Russie ; 9 aux États-Unis ; 14 en Asie, dont 12 au Japon, ce qui est considérable en comparaison avec les autres pays et par ailleurs ne manque pas d'interpeller quant aux possibilités futures de développement de nos collaborations avec les chercheurs chinois ; 4 en Tunisie et un quasi désert ailleurs (un seul abonné dans chacun des pays suivants : Australie, Israël, Nouvelle-Zélande, Tchad).

Cette répartition géographique contribue en grande partie à expliquer que la majorité des sociétaires ne se rendent pas souvent aux Assemblées générales de la Société (cf. graphique $n^{\circ} 4$ ). Dans l'enquête réalisée en 2005 , sur 128 réponses à cette question, 53 déclaraient n'avoir jamais participé à une Assemblée générale et 8 autres ne pas l'avoir fait dans les dix dernières années, soit près de la moitié au total. Dans l'autre moitié, ceux qui affirmaient être venus l'année précédente, dans les cinq dernières années ou dans les dix dernières années, la répartition est la suivante : 37 (environ $29 \%$ ) venus l'année précédente, 17 (13\%) dans les cinq dernières années, 13 (10\%) dans la dernière décennie.

8 Cette brève enquête, à comparer avec quelques autres plus anciennes ${ }^{5}$, reflète l'état actuel de la Société des études robespierristes, mais aussi, au-delà, l'état de la société française et de ses intellectuels, ainsi que de la recherche historique sur la période révolutionnaire. Il a ainsi existé, incontestablement, un temps où nombreux étaient ceux qui rejoignaient la Société des études robespierristes en raison de convictions politiques quant aux révolutions passées et à venir. Être « robespierriste» était alors compris par eux comme l'un des actes «militants » naturels ${ }^{6}$, d'autant que la querelle historiographique souvent réduite à un affrontement Soboul/Furet inclinait aussi à «monter au front " pour défendre certaines conceptions. De toute évidence, ce temps semble à bien des égards révolu ${ }^{7}$. On peut le déplorer ou non, mais, quoi qu'il en soit, ce ne sont pas les évolutions politiques de la France contemporaine qui à elles seules peuvent conditionner l'avenir de la recherche sur la Révolution française. Peut-être peuvent-elles même être un vecteur de renouveau? Des terrains de recherche sont encore en friche, d'autres doivent susciter de nouveaux travaux, et la Révolution est loin d'avoir perdu tout son attrait auprès des jeunes générations d'étudiants et de chercheurs. Tous ceux qui parmi nous enseignent cette période à l'Université et encadrent des recherches, masters et doctorats, peuvent l'attester. C'est là que réside l'avenir de la Société des études robespierristes. Elle a toujours pu et su se renouveler, y compris en des temps infiniment plus sombres, et je crois qu'elle y parviendra à nouveau.

\section{ANNEXES}




\section{Graphique $n^{\circ} 1$}

Structure par âge de la SER (2005)

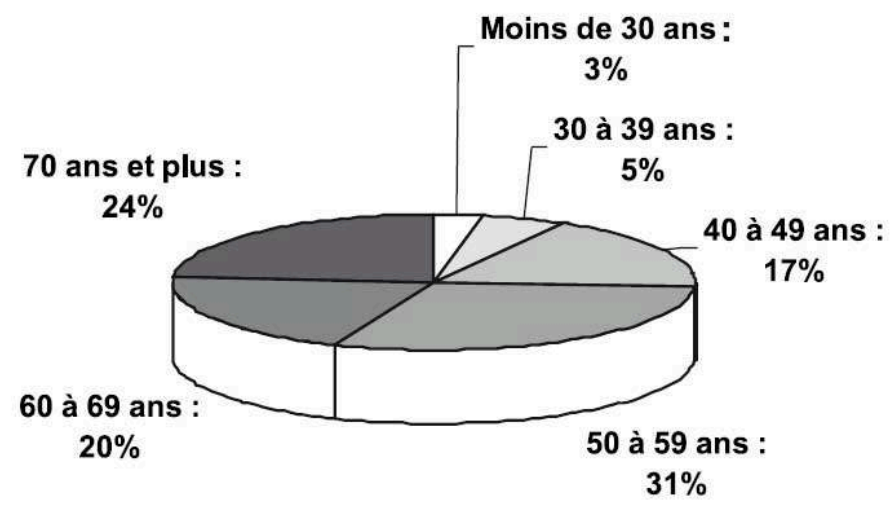

\section{Graphique $n^{\circ} 2$}

Dates d'adhésion à la SER (2005)

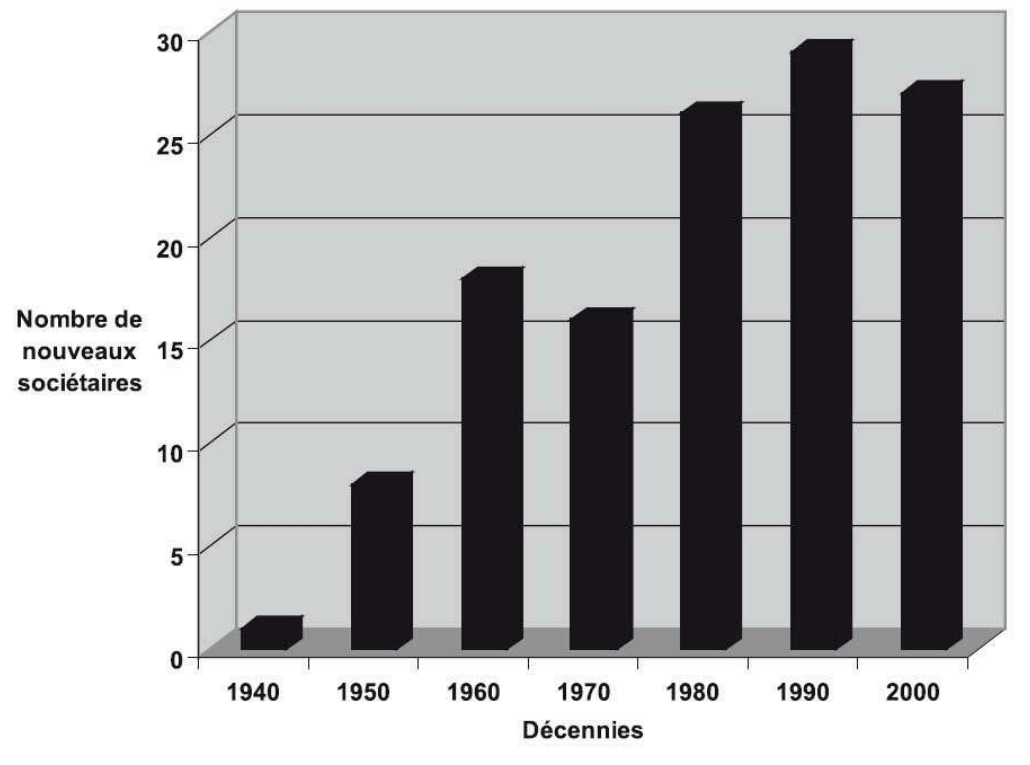




\section{Graphique $n^{\circ} 3$}

\section{Structure socio-professionnelle à la SER (2005)}

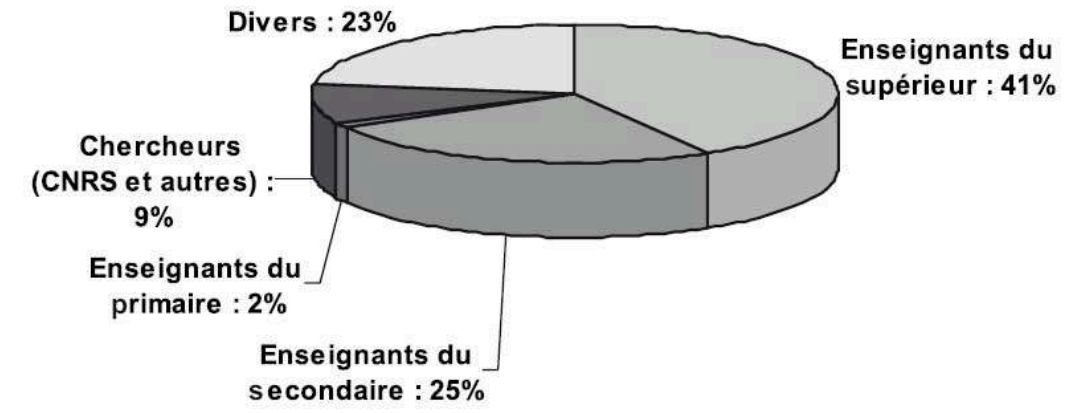



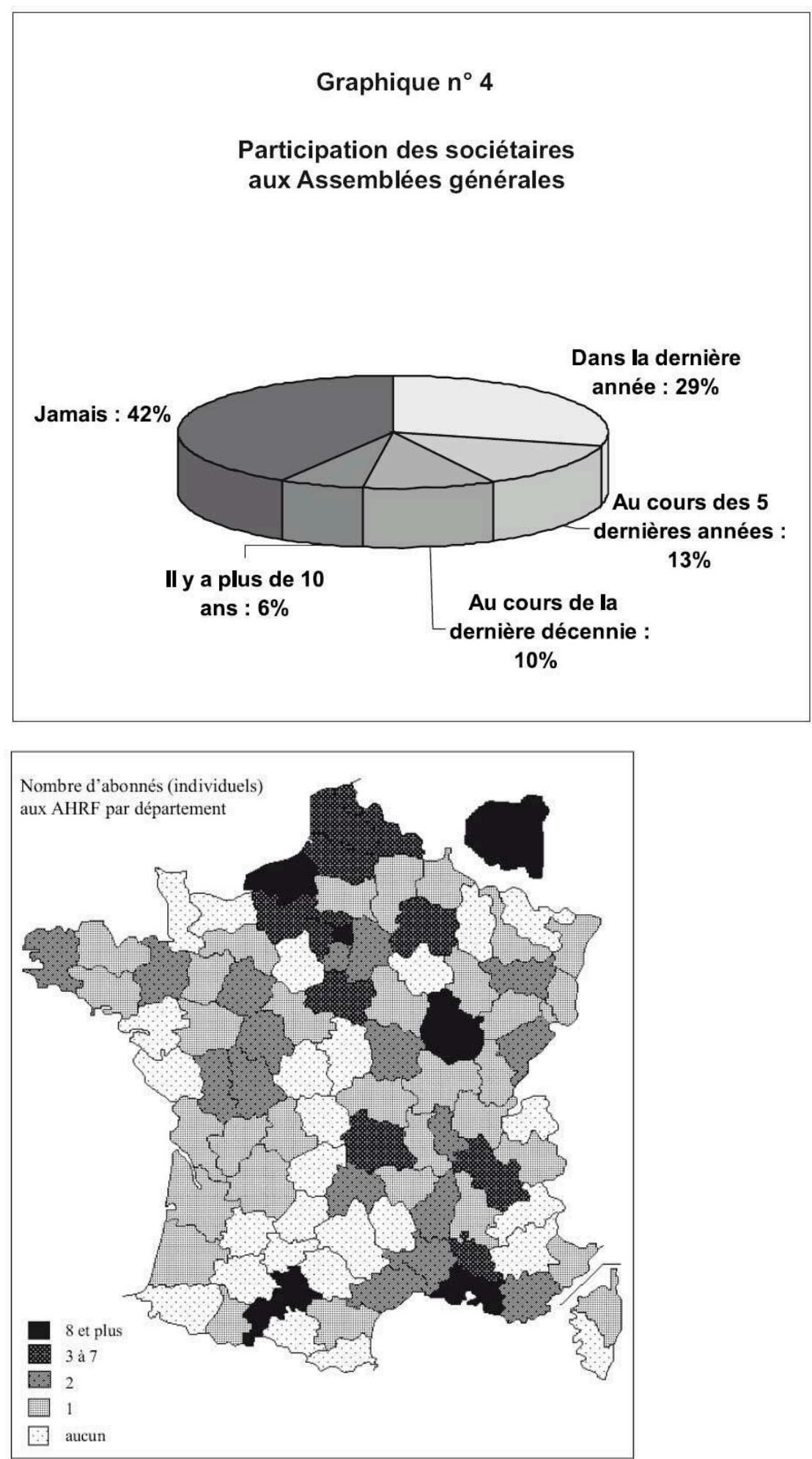

\section{NOTES}

1. Le nombre exact subissant quelques variations d'année en année, je préfère ici m'en tenir à un nombre approximatif. 
2. Les AHRF leur ont rendu hommage dans les numéros 2-2006 pour M. Slavin et 1-2007 pour R. Legrand.

3. Je ne donne évidemment ici que les noms de ceux qui ont bien voulu répondre à notre enquête. Il va de soi que plusieurs autres noms auraient sans doute pu être mentionnés pour ces années 1950.

4. Sur le graphique $\mathrm{n}^{\circ} 2$, la colonne des années 2000 ne correspond donc en réalité qu'à une demie décennie.

5. Voir notamment le $n^{\circ}$ 2-1991 des AHRF, p. 268-269. Les statistiques pour 1990 et 1991, donc aux lendemains du Bicentenaire, indiquaient alors environ 290 abonnés individuels en France et un peu plus de 100 à l'étranger, soit au final presque une centaine d'abonnés individuels de plus qu'aujourd'hui. Mais il serait intéressant aussi de rapprocher le nombre de thèses soutenues sur la période révolutionnaire dans les années qui ont immédiatement suivi 1989 et ce même nombre pour les années 1995-2005. Sans aucun doute le contraste serait-il évident.

6. Il suffirait sur ce point de poser aux sociétaires, notamment aux plus âgés, une autre question, digne des enquêtes américaines d'une triste époque et que nous n'avons bien sûr pas formulée lors de notre sondage : êtes ou avez-vous été membre du Parti communiste ou d'une organisation considérée comme proche de celui-ci?

7. Ce qui ne signifie en rien que les débats historiographiques soient apaisés. La seule relance récente d'une offensive ouvertement « contre-révolutionnaire ", dans la plus pure tradition d'un certain $\mathrm{XIX}^{\mathrm{E}}$ siècle, en témoigne avec ce Livre noir de la Révolution française aussi indigent dans ses apports que violent dans ses outrances.

\section{AUTEUR}

\section{MICHEL BIARD}

Directeur des AHRF Université de Rouen, GRHis 61, rue Lord Kitchener 76600 Le Havre

biard.m@wanadoo.fr 